\title{
The existence phase transition for scale invariant Poisson random fractal models.
}

\author{
Erik I. Broman*
}

January 10, 2019

\begin{abstract}
In this paper we study the existence phase transition of scale invariant random fractal models. We determine the exact value of the critical point of this phase transition for all models satisfying some weak assumptions. In addition, we show that for a large subclass, the fractal model is in the empty phase at the critical point. This subclass of models includes the scale invariant Poisson Boolean model and the Brownian loop soup. In contrast to earlier results in the literature, we do not need to restrict our attention to random fractal models generated by open sets.
\end{abstract}

\section{Introduction}

The questions studied in this paper are very much related to the classical problem of covering some fixed set by other randomly placed sets. The first paper dealing with this type of question was by Dvoretsky ([6]) who studied the problem of covering the unit circle by independently and uniformly placing arcs of given lengths. The study of such covering problems later developed in several different directions. One of the earliest papers dealing with questions similar to ours was by Shepp ([13]), who determined necessary and sufficient conditions for the real line to be covered by a Poisson process of random open intervals. The argument given in that paper relies on the fact that the problem is posed in dimension one, and cannot readily be generalized to higher dimensions. Many years later, Biermé and Estrade ([1]) and Broman, Jonasson and Tykesson ([3]), studied the scale invariant Poisson Boolean model (or fractal ball model) in general dimensions. However, in those papers it was assumed that that the balls generating the fractal set were open, and in addition, the arguments given relied on the simple geometry of these balls.

The purpose of this paper is two-fold. Firstly, we want to find arguments that do not rely on the sets generating the fractal being open. Secondly, we want to give a general result that is not restricted to the case where the generating sets have simple geometry (e.g. they are balls). Thereby, our results will cover cases such as the Brownian loop soup where the outer boundary is closed and does not have a simple geometry (see also Example 5.4).

*Department of Mathematics, Chalmers University of Technology and Gothenburg University, Sweden. E-mail: broman@chalmers.se. Supported by the Swedish Research Council 
Before stating exact results, we will begin by explaining the general setup. Let $\mathfrak{G}$ be the set of subsets of $\mathbb{R}^{d}$ with diameter strictly smaller than 1 . Furthermore, let $\mathcal{F}$ be some suitable $\sigma$-algebra on $\mathfrak{G}$. We consider infinite measures $\mu$ on $(\mathfrak{G}, \mathcal{F})$ which are semi scale invariant in the following sense. Assume that $\mathcal{D} \in \mathcal{F}$ is such that $\mu(\mathcal{D})<\infty$, and let $0<s<\infty$ be such that

$$
\mathcal{D}_{s}:=\left\{G \subset \mathbb{R}^{d}: G / s \in \mathcal{D}\right\}
$$

only contains sets of diameter strictly smaller than one. Then, semi scale invariance simply means that $\mu\left(\mathcal{D}_{s}\right)=\mu(\mathcal{D})$. We shall also require $\mu$ to be translational invariant in that $\mu(x+\mathcal{D})=\mu(\mathcal{D})$ for every $\mathcal{D} \in \mathcal{F}$ and $x \in \mathbb{R}^{d}$. Here of course, $x+\mathcal{D}=\{L \subset$ $\mathbb{R}^{d}: L=x+G$ for some $\left.G \in \mathcal{D}\right\}$.

We then let $\lambda \mu$ (where $0<\lambda<\infty$ is a parameter) be the intensity measure of a Poisson process $\Phi_{\lambda}(\mu)$ on $\mathfrak{G}$. Thus constructed, $\Phi_{\lambda}(\mu)$ is a (semi) scale and translation invariant random collection of bounded subsets of $\mathbb{R}^{d}$. This setup includes the scale invariant Poisson Boolean model (again, see [1] and [3]) and the Brownian loop soup introduced by Lawler and Werner in 9 .

We do not give details of how to construct general triplets $(\mathfrak{G}, \mathcal{F}, \mu)$ in this paper, but instead we refer the reader to the book [11] by Molchanov. Our viewpoint is rather that given such a triplet, the results of this paper will apply. We also point out that there are plenty of such triplets including the examples mentioned above and the ones listed later in this paper.

Throughout the rest of the paper, we shall make the following three natural assumptions on the measure $\mu$.

Assumption 1. Let $A \subset \mathbb{R}^{d}$ be bounded. Then, for any $\delta>0$ we have that

$$
\mu(G \in \mathfrak{G}: G \cap A \neq \emptyset, \operatorname{diam}(G) \geq \delta)<\infty .
$$

This assumption will make sure that the Poisson process never has infinitely many sets of diameter larger than some constant in any bounded region. Thus, Assumption 1 says that $\mu$ is locally finite.

For our second assumption, let $\mathcal{L}(\cdot)$ denote Lebesgue measure in $\mathbb{R}^{d}$ and let $\partial G$ denote the boundary of the set $G$.

Assumption 2. We have that $\mu(G \in \mathfrak{G}: \mathcal{L}(\partial G)>0)=0$.

This assumption makes sure that the boundary of the sets generating the fractal is not "too large".

Assumption 3. We have that $\mu(G \in \mathfrak{G}: \mathcal{L}(G)>0)>0$.

Let $\operatorname{Int}(G)$ denote the interior of a set $G$. Note that under Assumption 2 we have that $\mathcal{L}(G \cup \partial G)=\mathcal{L}(G)$, which means that if $\mathcal{L}(G)=0$ then $\operatorname{Int}(G \cup \partial G)=\emptyset$ and so $G$ is a nowhere dense set. Therefore, if $\mu$ is concentrated on sets such that $\mathcal{L}(G)=0$, it follows from Baire's category theorem that $\mathcal{C}(\lambda) \neq \emptyset$ for every $\lambda<\infty$. This explains the necessity of Assumption 3 (see further the remark after Theorem 1.1 below). 
Note also that by Assumptions 2 and 3 we have that $\mathcal{L}(\operatorname{Int}(G)) \geq \mathcal{L}(G)-\mathcal{L}(\partial G)=$ $\mathcal{L}(G)>0$, and so $\mu$ puts positive mass on sets $G$ with non-empty interiors.

It will be convenient to split the measure $\mu$ into two parts. Firstly, we define the measure $\mu_{p}$ by letting $\mu_{p}(\mathcal{A})=\mu(\{G \in \mathcal{A}: \mathcal{L}(G)>0\})$ for any $\mathcal{A} \in \mathcal{F}$, and secondly we define $\mu_{0}$ by letting $\mu_{0}(\mathcal{A})=\mu(\{G \in \mathcal{A}: \mathcal{L}(G)=0\})$. Obviously we then have that $\mu=\mu_{p}+\mu_{0}$. Although we will consider the case where $\mu_{0}$ is allowed to be non-trivial, we point out that the case where $\mu=\mu_{p}$ is somewhat easier to handle as it avoids some technical difficulties. See also the remark after the proof of Theorem 1.2.

Our random fractal is then defined by letting

$$
\mathcal{C}\left(\Phi_{\lambda}(\mu)\right):=\mathbb{R}^{d} \backslash \bigcup_{G \in \Phi_{\lambda}(\mu)} G,
$$

and we will write $\mathcal{C}(\lambda)$ or simply $\mathcal{C}$. It is not hard to prove (using Assumption 3 ) that for any $\lambda>0$ and any fixed $x \in \mathbb{R}^{d}$, we have that $\mathbb{P}(x \in \mathcal{C})=0$. We see that $\mathcal{C}$ is a (semi) scale invariant random fractal, and we will be concerned with various properties of $\mathcal{C}(\lambda)$ as $\lambda$ varies. It is useful to observe that by using a standard coupling argument, $\mathcal{C}(\lambda)$ is decreasing in $\lambda$.

Random fractal models exhibit several phase transitions (see for instance Dekking and Meester, [5]). The two most studied are the existence and the connectivity phase transitions, as we now explain. Define

$$
\lambda_{e}:=\inf \{\lambda>0: \mathbb{P}(\mathcal{C}(\lambda)=\emptyset)=1\} .
$$

Thus for $\lambda>\lambda_{e}, \mathcal{C}(\lambda)$ is almost surely empty, and we say that it is in the empty phase. Clearly, this is the same as saying that $\mathbb{R}^{d}$ is covered by $\bigcup_{G \in \Phi_{\lambda}(\mu)} G$. If on the other hand $\lambda<\lambda_{e}$, then $\mathbb{P}\left(\mathcal{C}(\lambda) \cap[0,1]^{d} \neq \emptyset\right)>0$. Thus, by tiling $\mathbb{R}^{d}$ with translates of $[0,1]^{d}$ and noting that $\mathcal{C}(\lambda) \cap B_{1}$ and $\mathcal{C}(\lambda) \cap B_{2}$ are independent whenever $B_{1}, B_{2} \subset \mathbb{R}^{d}$ are such that $d\left(B_{1}, B_{2}\right) \geq 1$, we conclude that $\mathbb{P}(\mathcal{C}(\lambda) \neq \emptyset)=1$. Hence, $\lambda_{e}$ is the critical point of the existence phase transition. Furthermore, we can define

$$
\lambda_{c}:=\sup \{\lambda>0: \mathbb{P}(\mathcal{C}(\lambda) \text { contains connected components larger than one point })=1\} .
$$

This means that if $\lambda>\lambda_{c}$, then $\mathcal{C}(\lambda)$ is almost surely totally disconnected. However, if $\lambda<\lambda_{c}$, then $\mathcal{C}(\lambda)$ will almost surely contain connected components (by the same tiling argument as before).

It is natural and interesting to ask what happens at the critical points of these phase transitions. In [2] it was proven that

$$
\mathbb{P}\left(\mathcal{C}\left(\lambda_{c}\right) \text { contains connected components larger than one point }\right)=1 \text {, }
$$

for any random fractal model satisfying Assumptions 1 and 3 . Therefore, at $\lambda_{c}$ the corresponding fractal is in the connected phase. In that respect, this phase transition is very well understood.

As explained, the existence phase transition is closely related to classical problems of random coverings, and we can now explain the results of [1] and [3] in terms of the terminology just introduced. In [1], the exact value of $\lambda_{e}$ was determined for the fractal 
ball model. In fact, their result was stronger than this, but it did not cover what happened at the critical value, i.e. whether $\mathbb{P}\left(\mathcal{C}\left(\lambda_{e}\right)=\emptyset\right)=0$ or 1 . This case was determined in [3] where it was proven that $\mathbb{P}\left(\mathcal{C}\left(\lambda_{e}\right)=\emptyset\right)=1$ for the particular case when $\mu$ is supported on open balls. In that paper, the corresponding result was also proven when $\mu$ is supported on open boxes.

Let

$$
\mathcal{A}_{l}:=\left\{G \in \mathfrak{G}: o \in G, 2^{-l} \leq \operatorname{diam}(G)<2^{-l+1}\right\},
$$

where $o$ denotes the origin in $\mathbb{R}^{d}$. It follows by scale invariance that $\mu\left(\mathcal{A}_{l}\right)=\mu\left(\mathcal{A}_{l+1}\right)$ for every $l \geq 1$.

Remark: In light of the definition of $\mathcal{D}_{s}$ in (1.1) above, it might be more natural to denote the left hand side of (1.3) by $\mathcal{A}_{2^{-l}}$. However, it would quickly be cumbersome to use this notation. Similar remarks apply throughout the paper.

Our first main result is the following.

Theorem 1.1. For any $\mu$ satisfying Assumptions 1, 2 and 3 we have that

$$
\lambda_{e}=\frac{d \log 2}{\mu\left(\mathcal{A}_{1}\right)} .
$$

Remarks: The use of $\mathcal{A}_{1}$ is somewhat arbitrary. Indeed, by scaling invariance we have that for any $k \geq 1$,

$$
\mu\left(G \in \mathfrak{G}: o \in G, 2^{-1} \leq \operatorname{diam}(G)<1\right)=k \mu\left(G \in \mathfrak{G}: o \in G, 2^{-1 / k} \leq \operatorname{diam}(G)<1\right) .
$$

As we shall see in Section 4, there is a canonical way of rewriting the expression for $\lambda_{e}$. Informally, this alternative expression involves the (quasi-)expected volume of sets of diameter exactly one. However, as this discussion is too long to fit in the introduction we defer it to Section 4 where the alternative expression appears in (4.5).

From alternative expressions for $\mu\left(\mathcal{A}_{1}\right)$ derived later in the paper (i.e. 4.4), it is easy to see that if Assumption 3 is not satisfied, (i.e. if $\mu(G \in \mathfrak{G}: \mathcal{L}(G)>0)=0$ ), then it follows that $\mu\left(\mathcal{A}_{1}\right)=0$. According to the discussion after the statement of Assumption 3, we will in this situation have that $\lambda_{e}=\infty$. If we then just interpret $d \log 2 / 0$ as being $\infty$, it is possible to drop Assumption 3 from the statement of Theorem 1.1.

It is natural to ask whether Theorem 1.1] still holds when Assumption 2 is not satisfied. We do not have any example showing that this is not the case.

We now turn to the behaviour of $\mathcal{C}(\lambda)$ at the critical point $\lambda_{e}$. For any set $G$, let $[\partial G]^{r}=\{x \in G: d(x, \partial G)<r\} \subset G$, so that $[\partial G]^{r}$ is the inner $r$-neighbourhood of $\partial G$. Our second main result is the following.

Theorem 1.2. If $\mu$ satisfies Assumptions 1, 2, 3 and the additional condition that

$$
\int_{\mathcal{A}_{1}} \int_{0}^{1} \frac{1}{r} \frac{\mathcal{L}\left([\partial G]^{r}\right)}{\mathcal{L}(G)} d r d \mu_{p}(G)<\infty
$$

then we have that $\mathcal{C}\left(\lambda_{e}\right)=\emptyset$ almost surely. 
Remark: The condition (1.4) in Theorem 1.2 might look like a technical assumption made purely for convenience. However, the class of models that satisfy (1.4) is very large and includes the fractal ball model and the fractal generated by the outer boundaries of the Brownian loop soup. As we will see, our examples of models that do not satisfy Assumption 2 or condition (1.4) will be somewhat contrived. This of course does not rule out the possibility of a naturally occurring example which does not satisfy our assumptions and conditions. Note also the occurrence of $\mu_{p}$ in (1.4) which guarantees that $\mathcal{L}(G)>0$ in the integrand.

The above mentioned examples will briefly be analysed in Section 5 (along with a third example) where we discuss what our main theorems imply for them. It is appropriate to mention the paper by Nacu and Werner ([12]), which deals with Hausdorff dimensions of the so-called Gasket of two-dimensional loop soups. In that paper a condition appears under the name "thin", which is a slightly stronger version of condition (1.4). We give the exact statement of this condition in (4.2). We note that condition (1.4), Assumption 2 and the condition of being thin all deal with the boundaries $\partial G$ of the sets used to generate the random fractal. It is therefore natural to ask how Assumption 2 and conditions (1.4), (4.2) relate to each other. The answer to this is given by the following proposition.

\section{Proposition 1.3.}

(i) If the measure $\mu$ is thin, then Assumption 2 and condition (1.4) are both satisfied.

(ii) If $\mu$ is supported on compact sets, then condition (1.4) implies Assumption 2, while for general measures $\mu$ this is not the case.

(iii) Assumption Q does not imply condition (1.4).

Remark: Assumption 1 is a fundamentally different type of condition than the ones we deal with in Proposition 1.3, and it is therefore not so natural to try to relate it to these other conditions. However, we add a short discussion on this subject after the proof of Proposition 1.3 .

We also note that the proof of Proposition 1.3 provides a concrete example of a measure $\mu$ not covered by our results. It will be a measure that does not satisfy Assumption 2 .

We will mention one more result. Assume momentarily that $\mu$ is supported on open sets, and that $\tilde{\mu}$ is the measure induced by taking the closure of these sets. We then have the following corollary of Theorems 1.1 and 1.2 .

Corollary 1.4. If $\mu$ satisfies Assumptions 1, 2 and [3, then the critical values for $\mu$ and $\tilde{\mu}$ are the same, i.e. $\lambda_{e}(\mu)=\lambda_{e}(\tilde{\mu})$.

In particular, Corollary 1.4 implies that the two fractal ball models generated by open balls and closed balls respectively, have the same critical value. Furthermore, it follows from Theorem 1.2 (see Example 5.2) that $\mathcal{C}\left(\lambda_{e}\right)=\emptyset$ for both of these variants.

The rest of the paper is organized as follows. In Section 2 we establish necessary notation and a preliminary result. In Section 3 we will prove Theorem 1.1 while in Section 4 we prove Theorem 1.2. In addition, Section 4 also contains the proof of Proposition 1.3 and the alternative expression for $\lambda_{e}$ mentioned above. This alternative expression will be used to prove Corollary [1.4. Finally, Section 5 contains the above mentioned examples. 


\section{Models and preliminary results}

As explained in the introduction, the measure $\mu$ is a measure on

$$
\mathfrak{G}:=\left\{G \subset \mathbb{R}^{d}: \operatorname{diam}(G)<1\right\},
$$

and $\Phi_{\lambda}=\Phi_{\lambda}(\mu)$ is a Poisson process on $\mathfrak{G}$ using $\lambda \mu$ as its intensity measure. Furthermore, we let

$$
\mathfrak{G}_{n}:=\left\{G \subset \mathbb{R}^{d}: 2^{-n} \leq \operatorname{diam}(G)<1\right\},
$$

and

$$
\Phi_{\lambda, n}:=\left\{G \in \Phi_{\lambda}(\mu): G \in \mathfrak{G}_{n}\right\},
$$

so that $\Phi_{\lambda, n}$ is a subset of the process $\Phi_{\lambda}$ containing sets of diameter larger than or equal to $2^{-n}$. Then, define

$$
\mathcal{C}_{n}:=\mathbb{R}^{d} \backslash \bigcup_{G \in \Phi_{\lambda, n}} G
$$

and note that $\mathcal{C}_{n} \supset \mathcal{C}_{n+1}$ for every $n$. Note also that by (1.2) $\mathcal{C}_{n} \downarrow \mathcal{C}$. For $m>n$, let

$$
\mathcal{C}_{m}^{n}:=\mathbb{R}^{d} \backslash \bigcup_{G \in \Phi_{\lambda, m} \backslash \Phi_{\lambda, n}} G
$$

so that $\mathcal{C}_{m}^{n} \cap \mathcal{C}_{n}=\mathcal{C}_{m}$, and $\mathcal{C}_{m}^{n}, \mathcal{C}_{n}$ are independent. Next, let

$$
\mathfrak{G}^{o}:=\{G \in \mathfrak{G}: o \in G\},
$$

and

$$
\mathfrak{G}_{n}^{o}:=\left\{G \in \mathfrak{G}_{n}: o \in G\right\},
$$

so that $\mathfrak{G}_{n}^{o}$ consists of the sets that have diameter larger than $2^{-n}$ while containing the origin. We observe from (1.3) that

$$
\mathfrak{G}_{n}^{o}=\bigcup_{l=1}^{n} \mathcal{A}_{l}
$$

and by scaling invariance we have that

$$
\mu\left(\mathfrak{G}_{n}^{o}\right)=\sum_{l=1}^{n} \mu\left(\mathcal{A}_{l}\right)=n \mu\left(\mathcal{A}_{1}\right) .
$$

Next, we let

$$
\mathcal{B}_{n}:=\left\{x+\left[0,2^{-n}\right]^{d}: x \in\left(2^{-n} \mathbb{Z}^{d}\right) \cap\left[0,1-2^{-n}\right]^{d}\right\},
$$

so that $\mathcal{B}_{n}$ consists of $2^{d n}$ closed boxes with non-overlapping interiors and $\bigcup_{B \in \mathcal{B}_{n}} B=$ $[0,1]^{d}$. We shall refer to a box $B \in \mathcal{B}_{n}$ as a level $n$ box. Furthermore, for any $A \subset \mathcal{B}_{n}$ we define $|A|$ to be the cardinality of $\left\{B \in \mathcal{B}_{n}: B \in A\right\}$. Then, let

$$
M_{n}:=\left\{B \in \mathcal{B}_{n}: \nexists G \in \Phi_{\lambda, n} \text { such that } B \subset G\right\}
$$


Of course, we could have used $\Phi_{\lambda}$ in the definition without anything being changed. Thus, $M_{n}$ is the set of level $n$ boxes which are not singly covered by a set in the Poisson process $\Phi_{\lambda, n}$. Furthermore, let

$$
m_{n}:=\left\{B \in \mathcal{B}_{n}: \nexists G \in \Phi_{\lambda, n} \text { such that } B \cap G \neq \emptyset\right\} .
$$

Hence, $m_{n}$ is the set of level $n$ boxes that are completely untouched by the sets of $\Phi_{\lambda, n}$. It is immediate that $m_{n} \subset M_{n}$ for every $n$. Let $D_{n}=D_{n}\left(\mathcal{C}_{n}\right)$ be a minimal (with respect to the number of boxes) collection of boxes in $\mathcal{B}_{n}$ such that

$$
\mathcal{C}_{n} \cap[0,1]^{d} \subset \bigcup_{B \in D_{n}} B
$$

Since a point $x \in \mathcal{C}_{n}$ in the intersection of two boxes $B_{1}$ and $B_{2}$ can be covered by either one of them, it follows that $D_{n}$ is not necessarily unique. If there is more than one way of choosing such a set $D_{n}$, we use some predetermined rule to pick one. Finally, we let $L_{n}:=\left|D_{n}\right|$.

A version of the following easy lemma appears in [3]. However, since the proof is short, we include it here for completeness.

Lemma 2.1. For any $\mu$ satisfying Assumption [3, if $\lambda>0$ is such that $\mathbb{P}(\mathcal{C}(\lambda) \neq \emptyset)=1$, then we must have that

$$
\mathbb{P}\left(\lim _{n \rightarrow \infty}\left|M_{n}\right|=\infty\right)>0 .
$$

Proof. First, any $B \in D_{n}$ contains a point which is in $\mathcal{C}_{n}(\lambda)$ and therefore it cannot be that $B$ is covered by a single set $G$ in the Poisson process $\Phi_{\lambda, n}$. Therefore, $B \in M_{n}$ and so $L_{n} \leq\left|M_{n}\right|$.

Secondly, observe that by definition of $D_{n}$ we have that

$$
\left(\mathcal{C}_{n} \cap[0,1]^{d}\right) \backslash \bigcup_{B \in D_{n}} B=\emptyset .
$$

Furthermore, we have that by Assumption 3 there exists some $\alpha=\alpha(\lambda) \in(0,1)$, such that

$$
\mathbb{P}\left(\mathcal{C}_{1} \cap[0,1]^{d}=\emptyset\right)=\alpha .
$$

By using the FKG inequality for Poisson processes together with the scaling invariance of the models and the fact that $D_{n}$ is measurable with respect to the $\sigma$-algebra generated by $\Phi_{\lambda, n}$, we conclude that

$$
\begin{aligned}
& \mathbb{P}\left(\mathcal{C}_{n+1} \cap[0,1]^{d}=\emptyset \mid \Phi_{\lambda, n}\right) \\
& \quad \geq \mathbb{P}\left(\bigcap_{B \in D_{n}}\left\{\mathcal{C}_{n+1}^{n} \cap B=\emptyset\right\} \mid \Phi_{\lambda, n}\right) \geq \prod_{B \in D_{n}} \mathbb{P}\left(\mathcal{C}_{n+1}^{n} \cap B=\emptyset\right)=\alpha^{L_{n}}>0 .
\end{aligned}
$$

Therefore, if there exists $L<\infty$ such that $L_{n} \leq L$ for infinitely many $n$, it follows by standard arguments that (2.1) implies that $\mathcal{C} \cap[0,1]^{d}=\emptyset$ almost surely. By countability, we conclude that if $\mathbb{P}(\mathcal{C} \neq \emptyset)=1$ we must have that $\mathbb{P}\left(\lim _{n \rightarrow \infty} L_{n}=\infty\right)>0$. Since $L_{n} \leq\left|M_{n}\right|$ the statement follows. 


\section{Proof of Theorem 1.1}

In this section, we will prove Theorem 1.1. We will do this by showing the two directions separately.

\subsection{Lower bound}

This subsection is devoted to proving that $\lambda_{e} \geq \frac{d \log 2}{\mu\left(\mathcal{A}_{1}\right)}$, and this is done in two steps. Firstly, Lemma 3.1 proves that for any $\lambda<\frac{d \log 2}{\mu\left(\mathcal{A}_{1}\right)}$ we must have that $\mathbb{E}\left[\left|m_{n}\right|\right] \rightarrow \infty$. Then, in Theorem 3.2 we use this to embed a supercritical branching process of boxes belonging to $\left(m_{n}\right)_{n=1}^{\infty}$ in such a way that $\mathcal{C}(\lambda) \cap[0,1]^{d} \neq \emptyset$ whenever the branching process survives. In fact, with minimal extra effort, we will obtain the stronger statement that for every $\epsilon>0$ we can embed a supercritical branching process with growth rate (see [10] Chapter 1) at least $2^{\left(d-\lambda \frac{\mu\left(\mathcal{A}_{1}\right)+\epsilon}{\log 2}\right)}$.

Lemma 3.1. For any $\lambda, \epsilon>0$ and fractal model satisfying Assumptions 1, 2 and 3 there exists $N=N(\epsilon)<\infty$ such that

$$
\mathbb{E}\left[\left|m_{n}\right|\right] \geq 2^{n\left(d-\lambda \frac{\mu\left(\mathcal{A}_{1}\right)+\epsilon}{\log 2}\right)},
$$

for every $n \geq N$.

Proof. Let $D(x, r)$ denote an open ball centered at $x$ with radius $r$. Obviously, the box $\left[0,2^{-n}\right]^{d}$ can be inscribed in an open ball of radius $d 2^{-n}$. Therefore, by translation invariance, we have that for any $B \in \mathcal{B}_{n}$,

$$
\begin{aligned}
& \mathbb{P}\left(B \in m_{n}\right) \geq \mathbb{P}\left(\nexists G \in \Phi_{\lambda, n}: D\left(o, d 2^{-n}\right) \cap G \neq \emptyset\right) \\
& \quad=\exp \left(-\lambda \mu\left(\left\{G \in \mathfrak{G}_{n}: D\left(o, d 2^{-n}\right) \cap G \neq \emptyset\right\}\right)\right) .
\end{aligned}
$$

Let

$$
E(G, r):=\{x: d(x, G)<r\}
$$

so that $E(G, r)$ is an enlargement of $G$. If $o \in G$ then trivially $D(o, r) \cap G \neq \emptyset$ and $o \in E(G, r)$. Furthermore, if $o \in G^{c}$, then since $D(o, r)$ and $E(G, r)$ are both open we see that $D(o, r) \cap G \neq \emptyset$ implies that $d(o, \partial G)<r$ and so $o \in E(G, r)$. Furthermore, the reverse statements are also true. Therefore,

$$
\begin{aligned}
& \mu\left(\left\{G \in \mathfrak{G}_{n}: D\left(o, d 2^{-n}\right) \cap G \neq \emptyset\right\}\right) \\
& \quad=\mu\left(\left\{G \in \mathfrak{G}_{n}: o \in E\left(G, d 2^{-n}\right)\right\}\right)=\int_{\mathfrak{G}_{n}} I\left(o \in E\left(G, d 2^{-n}\right)\right) d \mu(G) .
\end{aligned}
$$

Note that for any fixed point $x \in \mathbb{R}^{d}$ we must have that

$$
\begin{aligned}
-x & +\left\{G \in \mathfrak{G}_{n}: o \in E\left(G, d 2^{-n}\right), x \in G\right\} \\
& =\left\{L \in \mathfrak{G}_{n}: L=-x+G, o \in E\left(G, d 2^{-n}\right), x \in G\right\} \\
& =\left\{G \in \mathfrak{G}_{n}: o \in E\left(G+x, d 2^{-n}\right), x \in(G+x)\right\} .
\end{aligned}
$$


Recall that $\mathcal{L}(\cdot)$ denotes Lebesgue measure in $\mathbb{R}^{d}$ and recall also the notation $\mu=\mu_{p}+\mu_{0}$. At this point we will need to consider $\mu_{p}$ and $\mu_{0}$ separately. We will start with $\mu_{p}$ and note that by translation invariance we have that

$$
\begin{array}{rl}
\int_{\mathfrak{G}_{n}} & I\left(o \in E\left(G, d 2^{-n}\right)\right) d \mu_{p}(G) \\
& =\int_{\mathfrak{G}_{n}} I\left(o \in E\left(G, d 2^{-n}\right)\right) \frac{1}{\mathcal{L}(G)} \int_{\mathbb{R}^{d}} I(x \in G) d x d \mu_{p}(G) \\
& =\int_{\mathbb{R}^{d}} \int_{\mathfrak{G}_{n}} \frac{1}{\mathcal{L}(G)} I\left(o \in E\left(G, d 2^{-n}\right)\right) I(x \in G) d \mu_{p}(G) d x \\
& =\int_{\mathbb{R}^{d}} \int_{\mathfrak{G}_{n}} \frac{1}{\mathcal{L}(G+x)} I\left(o \in E\left(G+x, d 2^{-n}\right) I(x \in(G+x)) d \mu_{p}(G) d x\right. \\
= & \int_{\mathbb{R}^{d}} \int_{\mathfrak{G}_{n}} \frac{1}{\mathcal{L}(G)} I\left(o \in E\left(G, d 2^{-n}\right)+x\right) I(o \in G) d \mu_{p}(G) d x \\
= & \int_{\mathbb{R}^{d}} \int_{\mathfrak{G}_{n}} \frac{1}{\mathcal{L}(G)} I\left(-x \in E\left(G, d 2^{-n}\right)\right) I(o \in G) d \mu_{p}(G) d x \\
= & \int_{\mathfrak{G}_{n}} \frac{\mathcal{L}\left(E\left(G, d 2^{-n}\right)\right)}{\mathcal{L}(G)} I(o \in G) d \mu_{p}(G) \\
= & \int_{\mathfrak{G}_{n}^{o}} \frac{\mathcal{L}\left(E\left(G, d 2^{-n}\right)\right)}{\mathcal{L}(G)} d \mu_{p}(G)=\sum_{l=1}^{n} \int_{\mathcal{A}_{l}} \frac{\mathcal{L}\left(E\left(G, d 2^{-n}\right)\right)}{\mathcal{L}(G)} d \mu_{p}(G) .
\end{array}
$$

We make the observation that since by (3.2) and (3.3),

$$
\sum_{l=1}^{n} \int_{\mathcal{A}_{l}} \frac{\mathcal{L}\left(E\left(G, d 2^{-n}\right)\right)}{\mathcal{L}(G)} d \mu_{p}(G) \leq \mu\left(\left\{G \in \mathfrak{G}_{n}: \quad D\left(o, d 2^{-n}\right) \cap G \neq \emptyset\right\}\right),
$$

then the left hand side must be finite by Assumption 1. Then, let

$$
a_{l, n}:=\int_{\mathcal{A}_{l}} \frac{\mathcal{L}\left(E\left(G, d 2^{-n}\right)\right)}{\mathcal{L}(G)} d \mu_{p}(G),
$$

and observe that by elementary properties of Lebesgue measure we have that

$$
2^{d} \mathcal{L}\left(E\left(G, d 2^{-n}\right)\right)=\mathcal{L}\left(2 E\left(G, d 2^{-n}\right)\right)=\mathcal{L}\left(E\left(2 G, d 2^{-n+1}\right)\right) .
$$

Therefore,

$$
\begin{aligned}
a_{l, n} & =\int_{\mathcal{A}_{l}} \frac{\mathcal{L}\left(E\left(G, d 2^{-n}\right)\right)}{\mathcal{L}(G)} d \mu_{p}(G) \\
& =\int_{\mathcal{A}_{l}} \frac{\mathcal{L}\left(E\left(2 G, d 2^{-n+1}\right)\right)}{\mathcal{L}(2 G)} d \mu_{p}(G)=\int_{\mathcal{A}_{l-1}} \frac{\mathcal{L}\left(E\left(G, d 2^{-n+1}\right)\right)}{\mathcal{L}(G)} d \mu_{p}(G)=a_{l-1, n-1} .
\end{aligned}
$$

Here, the third equality follows since the measure $\mu$ is scale invariant, and the fact that for any $G \in \mathcal{A}_{l}$ we have that $2 G \in \mathcal{A}_{l-1}$.

By Assumption 2 we have that $\mu(G \in \mathfrak{G}: \mathcal{L}(\partial G)>0)=0$, and so

$$
a_{1, n}=\int_{\mathcal{A}_{1}} \frac{\mathcal{L}\left(E\left(G, d 2^{-n}\right)\right)}{\mathcal{L}(G)} I(\mathcal{L}(\partial G)=0) d \mu_{p}(G) .
$$


Furthermore, we have that

$$
\bigcap_{n=1}^{\infty} E\left(G, d 2^{-n}\right)=G \cup \partial G,
$$

and so by the monotone convergence theorem we conclude that

$$
\begin{gathered}
\lim _{n \rightarrow \infty} a_{1, n}=\int_{\mathcal{A}_{1}} \lim _{n \rightarrow \infty} \frac{\mathcal{L}\left(E\left(G, d 2^{-n}\right)\right)}{\mathcal{L}(G)} I(\mathcal{L}(\partial G)=0) d \mu_{p}(G) \\
=\int_{\mathcal{A}_{1}} \frac{\mathcal{L}(G \cup \partial G)}{\mathcal{L}(G \cup \partial G)} I(\mathcal{L}(\partial G)=0) d \mu_{p}(G)=\mu_{p}\left(\mathcal{A}_{1}\right) .
\end{gathered}
$$

By (3.4), and the elementary fact that $a_{1, n} \geq a_{1, n+1}$ for any $n$, there exists some $C_{\mu}<\infty$ such that $a_{1, n} \leq C_{\mu}$ for every $n \geq 1$. For any fixed $\epsilon>0$ it follows from (3.6) that there exists an $N_{1}<\infty$ such that $a_{1, n} \leq \mu\left(\mathcal{A}_{1}\right)+\epsilon / 3$ for every $n \geq N_{1}$. Furthermore, there exists $N \geq N_{1}$ such that

$$
\left(N-N_{1}\right)\left(\mu_{p}\left(\mathcal{A}_{1}\right)+\epsilon / 3\right)+C_{\mu} N_{1} \leq N\left(\mu_{p}\left(\mathcal{A}_{1}\right)+\epsilon / 2\right) .
$$

By (3.5) we have that $a_{l, n}=a_{l-1, n-1}=\cdots=a_{1, n-l+1}$, and so we see that for any $n \geq N$,

$$
\begin{aligned}
& \sum_{l=1}^{n} a_{l, n}=\sum_{l=1}^{n} a_{1, n-l+1}=\sum_{l=1}^{n} a_{1, l} \\
& \quad \leq \sum_{l=1}^{N_{1}} a_{1, l}+\sum_{l=N_{1}+1}^{n} a_{1, l} \leq \sum_{l=1}^{N_{1}} C_{\mu}+\sum_{l=N_{1}+1}^{n}\left(\mu_{p}\left(\mathcal{A}_{1}\right)+\epsilon / 3\right) \\
& \quad=C_{\mu} N_{1}+\left(n-N_{1}\right)\left(\mu_{p}\left(\mathcal{A}_{1}\right)+\epsilon / 3\right) \leq n\left(\mu_{p}\left(\mathcal{A}_{1}\right)+\epsilon / 2\right) .
\end{aligned}
$$

Combining (3.3), (3.5) and (3.7) we obtain

$$
\int_{\mathfrak{G}_{n}} I\left(o \in E\left(G, d 2^{-n}\right)\right) d \mu_{p}(G) \leq n\left(\mu_{p}\left(\mathcal{A}_{1}\right)+\epsilon / 2\right)
$$

for every $n \geq N$.

We are now ready to turn our attention to $\mu_{0}$. However, as this case is similar to our previous case we will be brief. We have that (with the interpretation that $\mathfrak{G}_{0}=\emptyset$ )

$$
\begin{array}{rl}
\int_{\mathfrak{G}_{n}} & I\left(o \in E\left(G, d 2^{-n}\right)\right) d \mu_{0}(G) \\
= & \sum_{l=1}^{n} \int_{\mathfrak{G}_{l} \backslash \mathfrak{G}_{l-1}} I\left(o \in E\left(G, d 2^{-n}\right)\right) d \mu_{0}(G)=\sum_{l=1}^{n} \int_{\mathfrak{G}_{1}} I\left(o \in E\left(G, d 2^{-n+l-1}\right)\right) d \mu_{0}(G) \\
= & \sum_{l=1}^{n} \int_{\mathfrak{G}_{1}} I\left(o \in E\left(G, d 2^{-n+l-1}\right)\right) \frac{1}{\mathcal{L}(E(G, 1))} \int_{\mathbb{R}^{d}} I(x \in E(G, 1)) d x d \mu_{0}(G) \\
= & \sum_{l=1}^{n} \int_{\mathfrak{G}_{1}} \frac{\mathcal{L}\left(E\left(G, d 2^{-n+l-1}\right)\right)}{\mathcal{L}(E(G, 1))} I(o \in E(G, 1)) d \mu_{0}(G) \\
= & \sum_{l=1}^{n} \int_{\mathfrak{G}_{1}} \frac{\mathcal{L}\left(E\left(G, d 2^{-l}\right)\right)}{\mathcal{L}(E(G, 1))} I(o \in E(G, 1)) d \mu_{0}(G) .
\end{array}
$$


Here we used the scale invariance in the second equality and then a calculation similar to (3.3). Next, note that by Assumption 1 and the monotone convergence theorem we have that

$$
\begin{aligned}
& \lim _{l \rightarrow \infty} \int_{\mathfrak{G}_{1}} \frac{\mathcal{L}\left(E\left(G, d 2^{-l}\right)\right)}{\mathcal{L}(E(G, 1))} I(o \in E(G, 1)) d \mu_{0}(G) \\
& \quad=\int_{\mathfrak{G}_{1}} \frac{\mathcal{L}(G \cup \partial G)}{\mathcal{L}(E(G, 1))} I(o \in E(G, 1)) d \mu_{0}(G)=0,
\end{aligned}
$$

since $\mathcal{L}(G \cup \partial G)=0$ on the support of $\mu_{0}$. Therefore, by taking $N$ perhaps even larger than before, we obtain that

$$
\int_{\mathfrak{G}_{n}} I\left(o \in E\left(G, d 2^{-n}\right)\right) d \mu_{0}(G) \leq n \epsilon / 2
$$

for every $n \geq N$. We note that (as in the above calculations)

$$
\mu_{0}\left(\mathcal{A}_{1}\right)=\int_{\mathfrak{G}_{1}} I(o \in G) d \mu_{0}(G) \leq \lim _{l \rightarrow \infty} \int_{\mathfrak{G}_{1}} I\left(o \in E\left(G, d 2^{-l}\right)\right) d \mu_{0}(G)=0 .
$$

Hence, $\mu\left(\mathcal{A}_{1}\right)=\mu_{p}\left(\mathcal{A}_{1}\right)+\mu_{0}\left(\mathcal{A}_{1}\right)=\mu_{p}\left(\mathcal{A}_{1}\right)$ and so by combining (3.1), (3.2), (3.8) and (3.9) we then see that for every $n \geq N$,

$$
\begin{aligned}
& \mathbb{E}\left[\left|m_{n}\right|\right] \geq 2^{d n} \exp \left(-\lambda \int_{\mathfrak{G}_{n}} I\left(o \in E\left(G, d 2^{-n}\right)\right) d \mu(G)\right) \\
& \quad=2^{d n} \exp \left(-\lambda \int_{\mathfrak{G}_{n}} I\left(o \in E\left(G, d 2^{-n}\right)\right) d \mu_{p}(G)-\lambda \int_{\mathfrak{G}_{n}} I\left(o \in E\left(G, d 2^{-n}\right)\right) d \mu_{0}(G)\right) \\
& \quad \geq 2^{d n} \exp \left(-\lambda n\left(\mu_{p}\left(\mathcal{A}_{1}\right)+\epsilon\right)\right)=2^{n\left(d-\lambda \frac{\mu\left(\mathcal{A}_{1}\right)+\epsilon}{\log 2}\right)}
\end{aligned}
$$

as desired.

Remark: Note the we do not really use Assumption 3 in the above proof. The only purpose of this assumption is to guarantee that $\mu\left(\mathcal{A}_{1}\right)=\mu_{p}\left(\mathcal{A}_{1}\right)>0$.

Let

$$
\mathfrak{G}_{l N}^{(l-1) N}:=\left\{G \in \mathfrak{G}: 2^{-l N} \leq \operatorname{diam}(G)<2^{-(l-1) N}\right\}
$$

and

$$
\Phi_{l N}^{(l-1) N}:=\left\{G \in \Phi_{\lambda}(\mu): G \in \mathfrak{G}_{l N}^{(l-1) N}\right\} .
$$

Theorem 3.2. For any fractal model satisfying Assumptions 1 , 20 and 3 we have that

$$
\lambda_{e} \geq \frac{d \log 2}{\mu\left(\mathcal{A}_{1}\right)} .
$$

Proof. The strategy is to embed the family tree of a supercritical branching process within the sequence $\left(m_{n}\right)_{n \geq 1}$. The boxes of this branching process will then be used to prove that $\mathcal{C}(\lambda) \cap[0,1]^{d} \neq \emptyset$ whenever the branching process survives.

Fix $\lambda<\frac{d \log 2}{\mu\left(\mathcal{A}_{1}\right)}$ and $\epsilon>0$ such that $\alpha:=d-\lambda \frac{\mu\left(\mathcal{A}_{1}\right)+\epsilon}{\log 2}>0$. The first step is to pick $N$ so that $\mathbb{E}\left[\left|m_{N}\right|\right] \geq 3^{d} 2^{\alpha N}$ which we can do by Lemma 3.1. Given the set $m_{N} \subset \mathcal{B}_{N}$, we then 
let $W_{1}=W_{1}\left(m_{N}\right)$ be a maximal subset such that $d\left(B_{i}, B_{j}\right) \geq 2^{-N}$ for every $B_{i}, B_{j} \in m_{N}$ with $i \neq j$. For definitiveness, we can assume that $W_{1}$ is picked by some predetermined rule. Assume also that $\left\{B_{1}^{1}, B_{2}^{1}, \ldots, B_{Z_{1}}^{1}\right\}$ is an enumeration of the boxes $B \in W_{1}$ where of course, $Z_{1}:=\left|W_{1}\right|$. We observe that $Z_{1} \geq m_{N} / 3^{d}$. This follows since any two level $N$ boxes $B, B^{\prime} \in \mathcal{B}_{N}$ which have no points in common (i.e. they are not neighbours), must be at distance at least $2^{-N}$ from each other. We see that

$$
\mathbb{E}\left[Z_{1}\right] \geq \frac{\mathbb{E}\left[\left|m_{N}\right|\right]}{3^{d}} \geq 2^{\alpha N}
$$

by our assumption on $N$. Obviously, $Z_{1} \leq\left|m_{N}\right|$ and $Z_{1}$ is the first generation of our branching process, while the set of boxes in $W_{1}$ is the first generation of the corresponding family tree. Finally, let

$$
\mathcal{W}_{1}:=\bigcup_{B \in W_{1}} B
$$

and observe that $\mathcal{W}_{1} \subset \mathcal{C}_{N}$.

We proceed with the construction of the second generation. For any fixed $B_{i}^{1} \in$ $\left\{B_{1}^{1}, \ldots, B_{Z_{1}}^{1}\right\}$, let

$$
\mathcal{B}_{2 N}\left(B_{i}^{1}\right)=\left\{B \in \mathcal{B}_{2 N}: B \subset B_{i}^{1}\right\}
$$

and let

$$
m_{2 N, N}^{i}:=\left\{B \in \mathcal{B}_{2 N}\left(B_{i}^{1}\right): \nexists G \in \Phi_{2 N}^{N} \text { such that } B \cap G \neq \emptyset\right\} .
$$

We note that a box $B \in m_{2 N, N}^{i}$ is untouched by any set (in $\Phi_{\lambda}$ ) of diameter between $2^{-2 N}$ and $2^{-N}$. In addition, since $B \subset \mathcal{W}_{1}$ it must in fact also be untouched by any set (in $\Phi_{\lambda}$ ) of diameter larger than $2^{-N}$ and so $B \subset \mathcal{C}_{2 N}$.

We now make two key observations. Firstly, since $d\left(B_{i}^{1}, B_{j}^{1}\right) \geq 2^{-N}$ whenever $i \neq j$, we have that

$$
\left\{G \in \mathfrak{G}_{2 N}^{N}: G \cap B_{i}^{1} \neq \emptyset\right\} \cap\left\{G \in \mathfrak{G}_{2 N}^{N}: G \cap B_{j}^{1} \neq \emptyset\right\}=\emptyset
$$

for any $i \neq j$. This of course follows since for any $G \in \mathfrak{G}_{2 N}^{N}$ we have that $\operatorname{diam}(G)<2^{-N}$. It follows that given the set $\left\{B_{1}^{1}, \ldots, B_{Z_{1}}^{1}\right\}, m_{2 N, N}^{1}, \ldots, m_{2 N, N}^{Z_{1}}$ are all independent.

Secondly, the random collection of boxes $m_{2 N, N}^{i}$ have the same distribution (after translation) as $m_{2 N, N}^{j}$ for any $i, j \in\left\{1, \ldots, Z_{1}\right\}$ such that $i \neq j$. Furthermore, $\left(m_{2 N, N}^{i}\right)_{2^{N}}$ (recall the notation in (1.1)) has the same distribution as $m_{N}$ (again after translation). Let $W_{2, i}$ be a maximal subset of $m_{2 N, N}^{i}$ (using the same rule as the one used for picking $\left.W_{1}\right)$ such that $d\left(B_{j_{1}}^{2}, B_{j_{2}}^{2}\right) \geq 2^{-2 N}$ for every $B_{j_{1}}^{2}, B_{j_{2}}^{2} \in m_{2 N, N}^{i}$ with $j_{1} \neq j_{2}$. Then, let $Z_{2, i}:=\left|W_{2, i}\right|$. It follows that the sequence $\left\{Z_{2, i}\right\}_{i=1}^{Z_{1}}$ is i.d.d. and that every $Z_{2, i}$ has the same distribution as $Z_{1}$.

Then, let $W_{2}=\cup_{i=1}^{Z_{1}} W_{2, i}, Z_{2}=\sum_{i=1}^{Z_{1}} Z_{2, i}=\left|W_{2}\right|$ and

$$
\mathcal{W}_{2}:=\bigcup_{B \in W_{2}} B
$$

We observe that $Z_{2} \leq \sum_{i=1}^{Z_{1}}\left|m_{2 N, N}^{i}\right| \leq\left|m_{2 N}\right|$, and that $\mathcal{W}_{2} \subset \mathcal{C}_{2 N}$. Thus, $Z_{2}$ is the number of individuals in the second generation of our branching process while the boxes of $W_{2}$ make up the second generation of the corresponding family tree. 
It is clear that we can proceed with the construction for further generations in essentially the same way. Of course, the general step in the construction is very similar to the steps above, so we shall be brief. Assume therefore that $\left(W_{l}, \mathcal{W}_{l}, Z_{l}\right)$ has been constructed. First, we let $\left\{B_{1}^{l}, \ldots, B_{Z_{l}}^{l}\right\}$ be an enumeration of the level $l N$-boxes in $W_{l}$. We think of $\left\{B_{1}^{l}, \ldots, B_{Z_{l}}^{l}\right\}$ as the offspring of generation $l$. Then, let

$$
\mathcal{B}_{(l+1) N}\left(B_{i}^{l}\right)=\left\{B \in \mathcal{B}_{(l+1) N}: B \subset B_{i}^{l}\right\}
$$

which are the level $(l+1) N$-boxes that sits inside $B_{i}^{l}$. Furthermore, we let

$$
m_{(l+1) N, l N}^{i}:=\left\{B \in \mathcal{B}_{(l+1) N}\left(B_{i}^{l}\right): \nexists G \in \Phi_{(l+1) N}^{l N} \text { such that } B \cap G \neq \emptyset\right\} .
$$

As before, the sequence $\left(m_{(l+1) N, l N}^{i}\right)_{1 \leq i \leq Z_{l}}$ consists of independent random variables. Then, we define $W_{l+1, i}$ for $i \in\left\{1, \ldots, Z_{l}\right\}$ as above and let $Z_{l+1, i}:=\left|W_{l+1, i}\right|$. Finally, we let $W_{l+1}=\cup_{i=1}^{Z_{l}} W_{l, i}, Z_{l+1}:=\sum_{i=1}^{Z_{l}} Z_{l+1, i}=\left|W_{l+1}\right|$ and

$$
\mathcal{W}_{l+1}:=\bigcup_{B \in W_{l}} B
$$

Again, $\mathcal{W}_{l+1} \subset \mathcal{C}_{(l+1) N}$.

The sequence $\left(Z_{l}\right)_{l \geq 1}$ describes a branching process with mean offspring distribution larger than $2^{\alpha N}$. Since this is a supercritical process, it follows by standard theory that

$$
\mathbb{P}\left(\lim _{l \rightarrow \infty} Z_{l}=\infty\right)>0
$$

On this event, we have that $\mathcal{W}_{l} \neq \emptyset$ for every $l$. Furthermore, the sets $\mathcal{W}_{l}$ are all compact and $\mathcal{W}_{l} \supset \mathcal{W}_{l+1}$ for every $l \geq 1$, and so

$$
\mathcal{C}=\bigcap_{n=1}^{\infty} \mathcal{C}_{n}=\bigcap_{l=1}^{\infty} \mathcal{C}_{l N} \supset \bigcap_{l=1}^{\infty} \mathcal{W}_{l} \neq \emptyset .
$$

Remark: In [3], the corresponding result for the fractal ball model was proved using a second moment method. That is, it was proved that for $\lambda<\frac{d \log 2}{\mu\left(\mathcal{A}_{1}\right)}$ (see also Example 5.2 in Section (5) there exists $c>0$ such that for every $n \geq 1$,

$$
\mathbb{P}\left(\left|m_{n}\right|>0\right) \geq \frac{\mathbb{E}\left[\left|m_{n}\right|\right]^{2}}{\mathbb{E}\left[\left|m_{n}\right|^{2}\right]} \geq c>0
$$

Using Fatou's lemma, this then implies that with positive probability, $\left|m_{n}\right|>0$ for every $n$, and so $\mathcal{C}_{n} \cap[0,1]^{d} \neq \emptyset$ for every $n$. Furthermore, in that paper the balls were open, and so $\mathcal{C}_{n} \cap[0,1]^{d}$ was compact. Therefore, one can conclude that $\mathcal{C} \cap[0,1]^{d} \neq \emptyset$. This part of the argument will not work in the more general setup of this paper. Indeed, if $\mathcal{C}_{n} \cap[0,1]^{d}$ is not compact, the conclusion that $\mathcal{C} \cap[0,1]^{d} \neq \emptyset$ does not follow. Observe also that in general,

$$
\bigcup_{B \in m_{n_{2}}} B \not \subset \bigcup_{B \in m_{n_{1}}} B
$$


whenever $n_{1}<n_{2}$. For instance, it is entirely possible that $m_{1}=\left\{\left[0,2^{-1}\right]^{d}\right\}$ while $m_{2}=\left\{\left[2^{-1}, 2^{-1}+2^{-2}\right]^{d}\right\}$. Therefore, the fact that $\left|m_{n}\right|>0$ for every $n$ does not directly imply that $\mathcal{C} \cap[0,1]^{d} \neq \emptyset$. Instead, we need to show the existence of nested, non-empty and closed subsets of $\mathcal{C}_{n} \cap[0,1]^{d}$, which is why we need the branching process construction above.

From the proof of Theorem 3.2 we conclude the following corollary which may be of independent interest.

Corollary 3.3. For fixed $\lambda<\frac{d \log 2}{\mu\left(\mathcal{A}_{1}\right)}$ and any $\epsilon>0$, we can with positive probability embed a branching process with growth rate at least

$$
2^{d-\lambda \frac{\mu\left(\mathcal{A}_{1}\right)+\epsilon}{\log 2}}
$$

within $\left(m_{n}\right)_{n \geq 1}$.

\subsection{Upper bound}

The purpose of this section is to prove that $\lambda_{e} \leq \frac{d \log 2}{\mu\left(\mathcal{A}_{1}\right)}$ which is then combined with Theorem 3.2 to prove Theorem 1.1 .

Theorem 3.4. For any fractal model satisfying Assumptions 1 , 2 and 3 we have that

$$
\lambda_{e} \leq \frac{d \log 2}{\mu\left(\mathcal{A}_{1}\right)}
$$

Proof. Start by noting that an open ball of radius $2^{-n-1}$ can be inscribed in the box $\left[0,2^{-n}\right]^{d}$. Therefore,

$$
\begin{aligned}
& \mathbb{P}\left(B \in M_{n}\right)=\mathbb{P}\left(\left[0,2^{-n}\right]^{d} \in M_{n}\right) \\
& \quad \leq \exp \left(-\lambda \mu\left(\left\{G \in \mathfrak{G}_{n}: D\left(o, 2^{-n-1}\right) \subset G\right\}\right)\right) .
\end{aligned}
$$

Then, let

$$
S(G, r):=\{x \in G: d(x, \partial G) \geq r\},
$$

so that $S(G, r)$ is a shrunken version of $G$. Obviously, $D(o, r) \subset G$ implies that $o \in G$ and $d(o, \partial G) \geq r$ so that $o \in S(G, r)$. It is also easy to see that the reverse implications are true. Thus,

$$
\begin{aligned}
& \mu\left(\left\{G \in \mathfrak{G}_{n}: D\left(o, 2^{-n-1}\right) \subset G\right\}\right)=\mu\left(\left\{G \in \mathfrak{G}_{n}: o \in S\left(G, 2^{-n-1}\right)\right\}\right) \\
& \quad=\mu_{p}\left(\left\{G \in \mathfrak{G}_{n}: o \in S\left(G, 2^{-n-1}\right)\right\}\right)=\int_{\mathfrak{G}_{n}} I\left(o \in S\left(G, 2^{-n-1}\right)\right) d \mu_{p}(G),
\end{aligned}
$$

where the second equality follows since any $G \in \mathfrak{G}_{n}$ such that $\mathcal{L}(G)=0$ must have an empty interior. Next, similar to the proof of Lemma 3.1. we have that

$$
\int_{\mathfrak{G}_{n}} I\left(o \in S\left(G, 2^{-n-1}\right)\right) d \mu_{p}(G)=\int_{\mathfrak{G}_{n}} \frac{\mathcal{L}\left(S\left(G, 2^{-n-1}\right)\right)}{\mathcal{L}(G)} I(o \in G) d \mu_{p}(G) .
$$


Combining (3.10), (3.11) and (3.12) we then get that

$$
\begin{aligned}
\mathbb{P}(B & \left.\in M_{n}\right) \leq \exp \left(-\lambda \int_{\mathfrak{G}_{n}} \frac{\mathcal{L}\left(S\left(G, 2^{-n-1}\right)\right)}{\mathcal{L}(G)} I(o \in G) d \mu_{p}(G)\right) \\
& =\exp \left(-\lambda \int_{\mathfrak{G}_{n}^{o}} \frac{\mathcal{L}\left(S\left(G, 2^{-n-1}\right)\right)}{\mathcal{L}(G)} d \mu_{p}(G)\right)=\exp \left(-\lambda \sum_{l=1}^{n} \int_{\mathcal{A}_{l}} \frac{\mathcal{L}\left(S\left(G, 2^{-n-1}\right)\right)}{\mathcal{L}(G)} d \mu_{p}(G)\right) .
\end{aligned}
$$

Let

$$
b_{l, n}:=\int_{\mathcal{A}_{l}} \frac{\mathcal{L}\left(S\left(G, 2^{-n-1}\right)\right)}{\mathcal{L}(G)} d \mu_{p}(G)
$$

and observe that $b_{l, n}=b_{l-1, n-1}$ in the same way that we proved (3.5). Thus, $b_{l, n}=\cdots=$ $b_{1, n-l+1}$ for $l \leq n$. Next, we have that

$$
\bigcup_{n=1}^{\infty} S\left(G, 2^{-n-1}\right)=G \backslash \partial G .
$$

By the monotone convergence theorem and using Assumption 2, we then get that

$$
\begin{aligned}
& \lim _{n \rightarrow \infty} b_{1, n}=\int_{\mathcal{A}_{1}} \lim _{n \rightarrow \infty} \frac{\mathcal{L}\left(S\left(G, 2^{-n-1}\right)\right)}{\mathcal{L}(G)} d \mu_{p}(G) \\
& \quad=\int_{\mathcal{A}_{1}} \frac{\mathcal{L}(G \backslash \partial G)}{\mathcal{L}(G)} d \mu_{p}(G)=\int_{\mathcal{A}_{1}} \frac{\mathcal{L}(G \backslash \partial G)}{\mathcal{L}(G)} I(\mathcal{L}(\partial G)=0) d \mu_{p}(G)=\mu_{p}\left(\mathcal{A}_{1}\right)=\mu\left(\mathcal{A}_{1}\right),
\end{aligned}
$$

where the last equality was shown in the proof of Lemma 3.1. We conclude that $b_{1, n} \uparrow$ $\mu\left(\mathcal{A}_{1}\right)$.

Consider now some $\lambda>\frac{d \log 2}{\mu\left(\mathcal{A}_{1}\right)}$ and let $\epsilon>0$ be such that also

$$
\lambda>\frac{d \log 2}{\mu\left(\mathcal{A}_{1}\right)-\epsilon} .
$$

There exists an $N_{1}<\infty$ such that $b_{1, n} \geq \mu\left(\mathcal{A}_{1}\right)-\epsilon / 2$ for every $n \geq N_{1}$. Furthermore, there exists $N \geq N_{1}$ such that

$$
\left(N-N_{1}\right)\left(\mu\left(\mathcal{A}_{1}\right)-\epsilon / 2\right) \geq N\left(\mu\left(\mathcal{A}_{1}\right)-\epsilon\right) .
$$

We then see that for every $n \geq N$,

$$
\begin{aligned}
& \sum_{l=1}^{n} b_{l, n}=\sum_{l=1}^{n} b_{1, n-l+1}=\sum_{l=1}^{n} b_{1, l} \geq \sum_{l=N_{1}+1}^{n} b_{1, l} \\
& \quad \geq \sum_{l=N_{1}+1}^{n}\left(\mu\left(\mathcal{A}_{1}\right)-\epsilon / 2\right)=\left(n-N_{1}\right)\left(\mu\left(\mathcal{A}_{1}\right)-\epsilon / 2\right) \geq n\left(\mu\left(\mathcal{A}_{1}\right)-\epsilon\right) .
\end{aligned}
$$

Using (3.13) and (3.15) we have that for every $n \geq N$,

$$
\begin{aligned}
& \mathbb{E}\left[\left|M_{n}\right|\right] \leq 2^{d n} \exp \left(-\lambda \sum_{l=1}^{n} \int_{\mathcal{A}_{l}} \frac{\mathcal{L}\left(S\left(G, 2^{-n-1}\right)\right)}{\mathcal{L}(G)} d \mu_{p}(G)\right) \\
& =\exp \left(n d \log 2-\lambda \sum_{l=1}^{n} b_{l, n}\right) \leq \exp \left(n d \log 2-\lambda n\left(\mu\left(\mathcal{A}_{1}\right)-\epsilon\right)\right) \\
& =\exp \left(n\left(d \log 2-\lambda\left(\mu\left(\mathcal{A}_{1}\right)-\epsilon\right)\right)\right) \longrightarrow 0,
\end{aligned}
$$


since $d \log 2-\lambda\left(\mu\left(\mathcal{A}_{1}\right)-\epsilon\right)<0$ by (3.14).

It follows that we must have that $\mathbb{P}\left(\lim _{n \rightarrow \infty}\left|M_{n}\right|=\infty\right)=0$, and so by Lemma 2.1 we can then conclude that

$$
\mathbb{P}\left(\mathcal{C}(\lambda) \cap[0,1]^{d}=\emptyset\right)=1,
$$

from which the statement follows.

Proof of Theorem 1.1. This is immediate from Theorems 3.2 and 3.4 .

\section{Proof of Theorem 1.2}

The purpose of this section is to prove Theorem 1.2 and also to discuss the connection between condition (1.4) and the notion of thin as introduced in [12. In addition, we shall derive an alternative expression for $\lambda_{e}$.

We start by proving Theorem 1.2 which basically just combines the proof of Theorem 3.4 with condition (1.4).

Proof of Theorem 1.2. Note that by the definition of $[\partial G]^{r}$, and since $\mu_{p}\left(\mathcal{A}_{1}\right)=\mu\left(\mathcal{A}_{1}\right)$,

$$
\begin{aligned}
& \mu\left(\mathcal{A}_{1}\right)-b_{1, l}=\int_{\mathcal{A}_{1}} 1-\frac{\mathcal{L}\left(S\left(G, 2^{-l-1}\right)\right)}{\mathcal{L}(G)} d \mu_{p}(G) \\
& =\int_{\mathcal{A}_{1}} \frac{\mathcal{L}\left(G \backslash S\left(G, 2^{-l-1}\right)\right)}{\mathcal{L}(G)} d \mu_{p}(G)=\int_{\mathcal{A}_{1}} \frac{\mathcal{L}\left([\partial G]^{2^{-l-1}}\right)}{\mathcal{L}(G)} d \mu_{p}(G) .
\end{aligned}
$$

Furthermore,

$$
\begin{aligned}
& \sum_{l=1}^{\infty} \int_{\mathcal{A}_{1}} \frac{\mathcal{L}\left([\partial G]^{2^{-l-1}}\right)}{\mathcal{L}(G)} d \mu_{p}(G) \leq \sum_{l=1}^{\infty} \int_{\mathcal{A}_{1}} \frac{\mathcal{L}\left([\partial G]^{2^{-l}}\right)}{\mathcal{L}(G)} d \mu_{p}(G) \\
& \quad=\int_{\mathcal{A}_{1}} \sum_{l=1}^{\infty} 2 \int_{2^{-l}}^{2^{-l+1}} \frac{1}{2^{-l+1}} \frac{\mathcal{L}\left([\partial G]^{2^{-l}}\right)}{\mathcal{L}(G)} d r d \mu_{p}(G) \leq 2 \int_{\mathcal{A}_{1}} \int_{0}^{1} \frac{1}{r} \frac{\mathcal{L}\left([\partial G]^{r}\right)}{\mathcal{L}(G)} d r d \mu_{p}(G)<\infty,
\end{aligned}
$$

where the last inequality is condition (1.4). Therefore, as in the proof of Theorem 3.4. we have that for $\lambda=\lambda_{e}$

$$
\begin{aligned}
\mathbb{E}\left[\left|M_{n}\right|\right] \leq \exp \left(d n \log 2-\lambda_{e} \sum_{l=1}^{n} b_{1, l}\right) \\
=\exp \left(\frac{d \log 2}{\mu\left(\mathcal{A}_{1}\right)}\left(n \mu\left(\mathcal{A}_{1}\right)-\sum_{l=1}^{n} b_{1, l}\right)\right) \\
=\exp \left(\frac{d \log 2}{\mu\left(\mathcal{A}_{1}\right)} \sum_{l=1}^{n} \int_{\mathcal{A}_{1}} \frac{\mathcal{L}\left([\partial G]^{-l-1}\right)}{\mathcal{L}(G)} d \mu_{p}(G)\right) \\
\quad \leq \exp \left(\frac{d \log 2}{\mu\left(\mathcal{A}_{1}\right)} \sum_{l=1}^{\infty} \int_{\mathcal{A}_{1}} \frac{\mathcal{L}\left([\partial G]^{2-l-1}\right)}{\mathcal{L}(G)} d \mu_{p}(G)\right)<\infty .
\end{aligned}
$$


We conclude that $\lim \sup _{n \rightarrow \infty} \mathbb{E}\left[\left|M_{n}\right|\right]<\infty$, and the statement follows by using Lemma 2.1 as before.

Remark: It is clear from the proofs of Theorems 1.1 and 1.2, that for a fractal model $\mu$ satisfying Assumptions 1, 2 and 3, $\mu_{0}$ is irrelevant when it comes to the question of covering subsets of $\mathbb{R}^{d}$. However, this is not apriori obvious, which is why we chose not to restrict our attention to measures $\mu$ such that $\mu=\mu_{p}$.

In order to proceed, we will consider a particular way of constructing scale invariant measures $\mu$. We shall be somewhat informal, the missing details can be found in [12]. We note that the models considered in [12] do not have a cutoff as we have imposed on $\mu$ here. Instead, they consider the model in a bounded domain $D$ where any set $G \in \Phi_{\lambda}(\mu)$ such that $G \cap\left(\partial D \cup D^{c}\right) \neq \emptyset$ is removed. However, this has little impact on the construction. Let $L(G)$ denote the center of mass of $G$. This creates a sort of "anchor" for the set $G$, and in fact any such anchor would do.

Let $\nu_{o}$ be a finite measure on sets $H \subset \mathbb{R}^{d}$ such that $\operatorname{diam}(H)=1$ and $L(H)=o$, and consider the product measure

$$
\nu_{o} \times \rho^{-(d+1)} d \rho \times \mathcal{L}(d x) .
$$

Using the transformation $G=\rho H+x$ we can define a scale invariant measure $\mu$ with cutoff 1 , through

$$
\int F(G) d \mu(G):=\int_{\mathbb{R}^{d}} \int_{0}^{1} \int F(\rho H+x) d \nu_{o} \times \rho^{-(d+1)} d \rho \times \mathcal{L}(d x),
$$

where $F$ is in some suitable class of functions. Furthermore, any scale invariant measure $\mu$ can be constructed in such a way (see again [12]). There is a tiny difference in that we assume that $\mu$ is a measure on $\left\{G \subset \mathbb{R}^{d}: \operatorname{diam}(G)<1\right\}$ rather than $\left\{G \subset \mathbb{R}^{d}\right.$ : $\operatorname{diam}(G) \leq 1\}$. However, this is only a technical nuisance. The condition of $\mu$ being thin is defined in [12] to mean that

$$
\int_{0}^{1} \frac{1}{r} \int \mathcal{L}(E(H, r) \backslash S(H, r)) d \nu_{o}(H) d r<\infty .
$$

We now turn to the proof of Proposition 1.3 which relates Assumption 2 with conditions (1.4) and (4.2).

Proof of Proposition 1.3. We start by proving $(i)$. If $\mu$ is thin, it follows from (4.2) that $\int \mathcal{L}(\partial H) d \nu_{o}(H)=0$. Therefore $\nu_{o}$ is supported on sets such that $\mathcal{L}(\partial H)=0$. It follows that this is also the case for the measure $\mu$, and so Assumption 2 holds. 
In order to show that $\mu$ being thin implies condition (1.4) we observe that

$$
\begin{aligned}
\int_{\mathcal{A}_{1}} & \frac{\mathcal{L}\left([\partial G]^{r}\right)}{\mathcal{L}(G)} d \mu_{p}(G)=\int_{\mathcal{A}_{1}} \frac{\mathcal{L}\left([\partial G]^{r}\right)}{\mathcal{L}(G)} I(\mathcal{L}(G)>0) d \mu(G) \\
= & \int_{1 / 2}^{1} \int_{\mathbb{R}^{d}} \int \frac{\mathcal{L}((\rho H+x) \backslash S(\rho H+x, r))}{\mathcal{L}(\rho H+x)} I(o \in \rho H+x) I(\mathcal{L}(H)>0) \frac{1}{\rho^{d+1}} d \nu_{o}(H) \mathcal{L}(d x) d \rho \\
= & \int_{1 / 2}^{1} \int_{\mathbb{R}^{d}} \int \frac{\mathcal{L}(\rho H \backslash S(\rho H, r))}{\mathcal{L}(\rho H)} I(o \in \rho H+x) I(\mathcal{L}(H)>0) \frac{1}{\rho^{d+1}} d \nu_{o}(H) \mathcal{L}(d x) d \rho \\
= & \int_{1 / 2}^{1} \int \frac{\mathcal{L}(H \backslash S(H, r / \rho))}{\mathcal{L}(H)} \mathcal{L}(\rho H) I(\mathcal{L}(H)>0) \frac{1}{\rho^{d+1}} d \nu_{o}(H) d \rho \\
= & \int_{1 / 2}^{1} \int \mathcal{L}\left([\partial H]^{r / \rho}\right) \frac{1}{\rho} d \nu_{o}(H) d \rho \leq \log 2 \int \mathcal{L}\left([\partial H]^{2 r}\right) d \nu_{o}(H) .
\end{aligned}
$$

Thus,

$$
\begin{aligned}
\int_{\mathcal{A}_{1}} & \int_{0}^{1} \frac{1}{r} \frac{\mathcal{L}\left([\partial G]^{r}\right)}{\mathcal{L}(G)} d r d \mu_{p}(G) \leq \log 2 \int_{0}^{1} \frac{1}{r} \int \mathcal{L}\left([\partial H]^{2 r}\right) d \nu_{o}(H) d r \\
\leq & C+\log 2 \int_{0}^{1 / 2} \frac{1}{r} \int \mathcal{L}\left([\partial H]^{2 r}\right) d \nu_{o}(H) d r \\
& =C+\log 2 \int_{0}^{1} \frac{1}{r} \int \mathcal{L}\left([\partial H]^{r}\right) d \nu_{o}(H) d r \\
& \leq C+\log 2 \int_{0}^{1} \frac{1}{r} \int \mathcal{L}(E(H, r) \backslash S(H, r)) d \nu_{o}(H) d r
\end{aligned}
$$

and so we see that (4.2) indeed implies condition (1.4).

We now turn to $(\mathrm{ii})$. By a slight adjustment we can reverse the inequality in (4.3). Furthermore, if the measure $\mu$ is supported on compact sets, then $[\partial H]^{r} \supset \partial H$ for every $r \geq 0$, and so

$$
\int_{\mathcal{A}_{1}} \frac{\mathcal{L}\left([\partial G]^{r}\right)}{\mathcal{L}(G)} d \mu_{p}(G) \geq \log 2 \int \mathcal{L}\left([\partial H]^{r}\right) d \nu_{o}(H) \geq \log 2 \int \mathcal{L}(\partial H) d \nu_{o}(H) .
$$

Hence,

$$
\log 2 \int_{0}^{1} \frac{1}{r} \int \mathcal{L}(\partial H) d \nu_{o}(H) d r \leq \int_{0}^{1} \frac{1}{r} \int_{\mathcal{A}_{1}} \frac{\mathcal{L}\left([\partial G]^{r}\right)}{\mathcal{L}(G)} d \mu_{p}(G) d r,
$$

and so if condition (1.4) holds, we must have that $\int \mathcal{L}(\partial H) d \nu_{o}(H)=0$, which implies Assumption 2 ,

In order to prove that condition (1.4) does not imply Assumption 2 in general, we will construct a counterexample. Let $x_{1}, x_{2}, \ldots$ be an enumeration of the rational points in $[0,1]^{d}$, where $d \geq 2$, and let $\operatorname{Box}(x, r)$ denote an open box centred at $x$ with side length $r$. Then, we define

$$
H:=\bigcup_{k=1}^{\infty} \operatorname{Box}\left(x_{k}, 2^{-k-1}\right) .
$$

We see that $H$ is open and that $\mathcal{L}(H) \leq 2^{-d}$. We pick our measure $\nu_{o}$ as being unit mass on the set $H$. Obviously $\operatorname{diam}(H)>1$, but this is easily rectified and left for the reader. 
We have that $\partial H \supset[0,1]^{d} \backslash H$ so that $\mathcal{L}(\partial H) \geq 1-2^{-d}$ and so Assumption 2 cannot hold for this choice of $H$.

Observe that

$$
\mathcal{L}\left([\partial \operatorname{Box}(x, s)]^{r}\right)= \begin{cases}s^{d} & \text { if } s \leq 2 r \\ s^{d}-(s-2 r)^{d} & \text { if } s>2 r\end{cases}
$$

Observe also that since the boxes $\operatorname{Box}\left(x_{k}, 2^{-k-1}\right)$ and $\operatorname{Box}\left(x_{l}, 2^{-l-1}\right)$ can overlap for $k \neq l$, there exists some $c=c(d)<\infty$ independent of $r$ and such that

$$
\begin{aligned}
& \mathcal{L}\left([\partial H]^{r}\right) \leq \sum_{k=1}^{\infty} \mathcal{L}\left(\left[\partial \operatorname{Box}\left(x_{k}, 2^{-k-1}\right)\right]^{r}\right) \\
& \quad \leq \sum_{k=1}^{\left\lceil-\log _{2} r\right\rceil-3} 2^{-d k-d}-\left(2^{-k-1}-2 r\right)^{d}+\sum_{k=\left\lceil-\log _{2} r\right\rceil-2}^{\infty} 2^{-d k-d} \\
& \quad \leq \frac{c r}{2}+\sum_{k=1}^{\left\lceil-\log _{2} r\right\rceil-3} 2^{-d k-d}\left(1-\left(1-r 2^{k+2}\right)^{d}\right) \leq \frac{c r}{2}+\sum_{k=1}^{\left\lceil-\log _{2} r\right\rceil-3} 2^{-d k-d} d r 2^{k+2} \leq c r,
\end{aligned}
$$

where we use that $d \geq 2$. Furthermore, we also used that if $k \leq\left\lceil-\log _{2} r\right\rceil-3$ then $r 2^{k+2} \leq r 2^{\left\lceil-\log _{2} r\right\rceil-1} \leq 1$ and that $1-(1-x)^{d} \leq d x$ whenever $0 \leq x \leq 1$. Obviously, we here have that $\mu=\mu_{p}$ and using (4.3) we see that

$$
\int_{0}^{1} \frac{1}{r} \int_{\mathcal{A}_{1}} \frac{\mathcal{L}\left([\partial G]^{r}\right)}{\mathcal{L}(G)} d \mu_{p}(G) d r \leq \log 2 \int_{0}^{1} \frac{1}{r} \int \mathcal{L}\left([\partial H]^{2 r}\right) d \nu_{o}(H)<\infty
$$

and so condition (1.4) holds.

Finally, we prove (iii) by constructing an example satisfying Assumption 2 but not condition (1.4). This construction will be done sequentially, i.e. we will construct closed sets $K_{1}, K_{2}, \ldots \subset[0,1]^{d}$ and then define $H:=\left(\cap_{n=1}^{\infty} K_{n}\right)^{c}$. Every set $K_{n}$ will be the union of a set of boxes $\mathcal{K}_{n} \subset \mathcal{B}_{n}$ so that by definition of $\mathcal{B}_{n}$,

$$
K_{n}:=\bigcup_{B \in \mathcal{K}_{n}} B \subset[0,1]^{d} .
$$

Let $\mathcal{K}_{1}=\mathcal{B}_{1}$ so that $K_{1}=[0,1]^{d}$. Then, let $\mathcal{K}_{2}$ be the set of boxes $B \in \mathcal{B}_{2}$ such that $B=x+\left[0,2^{-2}\right]^{d}$ where $x_{1}+\cdots+x_{d}$ is an even number. Note that $\mathcal{K}_{2}$ consists of $2^{2 d} / 2$ boxes in a ( $d$-dimensional) chequerboard pattern. In general, let $\mathcal{K}_{n+1}$ consist of $\left\lceil 2^{d(n+1)} /(n+1)\right\rceil$ boxes of $\mathcal{B}_{n+1}$, picked so that $K_{n+1} \subset K_{n}$, and such that for any $B \in \mathcal{K}_{n}$, there exists $B^{\prime} \in \mathcal{K}_{n+1}$ such that $B^{\prime} \subset B$. This condition makes sure that the box $B \in \mathcal{K}_{n}$ is not removed in its entirety. It is straightforward to see that this is possible as we now (somewhat informally) explain. Observe that the set $K_{n}$ can be written as a union of $2^{d}\left\lceil 2^{d n} / n\right\rceil$ boxes from $\mathcal{B}_{n+1}$. Therefore, the number of boxes that needs to be deleted in order to create $K_{n+1}$ can be bounded by

$2^{d}\left\lceil 2^{d n} / n\right\rceil-\left\lceil 2^{d(n+1)} /(n+1)\right\rceil \leq 2^{d(n+1)}\left(\frac{1}{n}-\frac{1}{n+1}\right)+1=\frac{2^{d(n+1)}}{n(n+1)}+1 \leq \frac{1}{2} 2^{d}\left\lceil 2^{d n} / n\right\rceil$, 
where the last inequality holds for $n \geq 2$ and $d \geq 2$. However, a similar calculation for $n=1$ shows that for any $d \geq 2$ and any $n \geq 1$,

$$
2^{d}\left\lceil 2^{d n} / n\right\rceil-\left\lceil 2^{d(n+1)} /(n+1)\right\rceil \leq \frac{1}{2} 2^{d}\left\lceil 2^{d n} / n\right\rceil .
$$

Therefore, in every step of the construction, at least half of the boxes are kept and so it is always possible to keep at least one level $(n+1)$ subbox of $B \in \mathcal{K}_{n}$.

We then note that since $H^{c}=\bigcap_{n=1}^{\infty} K_{n}$ is closed, we must have that

$$
\mathcal{L}(\partial H)=\mathcal{L}\left(H^{c}\right)=\lim _{n \rightarrow \infty} \mathcal{L}\left(K_{n}\right)=\lim _{n \rightarrow \infty}\left\lceil 2^{d n} / n\right\rceil 2^{-d n}=0,
$$

and so Assumption 2 is satisfied. We also note that for every $B \in \mathcal{K}_{n}$ there exists $x \in H^{c}$ such that $x \in B$. This follows from the constraint in the construction of $\mathcal{K}_{n+1}$ from $\mathcal{K}_{n}$ which implies that $B \cap K_{n} \neq \emptyset$ for every $n \geq 1$, and so $B \cap H^{c} \neq \emptyset$ by compactness. This in turn implies that $d\left(y, H^{c}\right) \leq \sqrt{d} 2^{-n}$ for every $y \in K_{n}$ and so $[\partial H]^{\sqrt{d} 2^{-n}} \supset K_{n} \backslash H^{c}$ for every $n \geq 1$. Thus, for $\sqrt{d} 2^{-n} \leq r \leq \sqrt{d} 2^{-n+1}$

$$
\mathcal{L}\left([\partial H]^{r}\right) \geq \mathcal{L}\left([\partial H]^{\sqrt{d} 2^{-n}}\right) \geq \mathcal{L}\left(K_{n} \backslash H^{c}\right)=\mathcal{L}\left(K_{n}\right)=\left\lceil 2^{d n} / n\right\rceil 2^{-d n} \geq 1 / n .
$$

Therefore, by letting $\nu_{o}$ be unit mass on $H$ we see that

$$
\int_{0}^{1} \frac{1}{r} \int \mathcal{L}\left([\partial H]^{r}\right) d \nu_{o}(H) d r \geq \sum_{n=d}^{\infty} \int_{\sqrt{d} 2^{-n}}^{\sqrt{d} 2^{-n+1}} \frac{1}{\sqrt{d} 2^{-n+1}} \frac{1}{n} d r=\sum_{n=d}^{\infty} \frac{1}{2 n}=\infty
$$

and so condition (1.4) is not satisfied.

Remarks: Informally, the example in part $(i i)$ of Proposition 1.3 works since the the set $H$ has a small inner boundary but a large outer boundary.

Similar to (4.3), one obtains that

$$
\begin{aligned}
\mu(G & : G \cap D(o, 1) \neq \emptyset, \delta \leq \operatorname{diam}(G) \leq 1) \\
& =\int_{\delta}^{1} \int_{\mathbb{R}^{d}} \int I(o \in E(\rho H+x, 1)) \frac{1}{\rho^{d+1}} d \nu_{o}(H) \mathcal{L}(d x) d \rho \\
& =\int_{\delta}^{1} \int \mathcal{L}(E(H, 1 / \rho)) \frac{1}{\rho} d \nu_{o}(H) d \rho,
\end{aligned}
$$

which is finite whenever $\nu_{o}$ is finite. Thus, the finiteness of $\nu_{o}$ is equivalent to Assumption 1. However, if we allow ourselves to consider also infinite measures $\nu_{o}$, it is possible to find an example so that condition (1.4) is satisfied while Assumption 1 is not. We will give an informal description of how this is done. Let $\nu_{o}$ be a measure on sets $[0,1] \times[0, h]$ given by $\nu_{o}(\mathcal{L}(H) \geq l)=\int_{l}^{1} h^{-3 / 2} d h$. Obviously, $\nu_{o}$ is then an infinite measure. However, we see that

$$
\int \mathcal{L}\left([\partial H]^{r}\right) d \nu_{o}(H) \leq \int_{0}^{2 r} h^{-1 / 2} d h+4 \int_{2 r}^{1} r h^{-3 / 2} d h \leq C \sqrt{r},
$$

and as above we get that condition (1.4) is satisfied. 
We now proceed to find the canonical expression for $\lambda_{e}$ mentioned in the Introduction. To that end, observe that as above,

$$
\begin{aligned}
\mu\left(\mathcal{A}_{1}\right) & =\int_{1 / 2}^{1} \int_{\mathbb{R}^{d}} \int I(o \in \rho H+x) \frac{1}{\rho^{d+1}} d \nu_{o}(H) \mathcal{L}(d x) d \rho \\
= & \int_{1 / 2}^{1} \int \mathcal{L}(H) \frac{1}{\rho} d \nu_{o}(H) d \rho=\log 2 \int \mathcal{L}(H) d \nu_{o}(H)=\nu_{o}(\mathcal{L}(H)) \log 2 .
\end{aligned}
$$

Thus we may write $\lambda_{e}$ as

$$
\lambda_{e}=\frac{d}{\nu_{o}(\mathcal{L}(H))} .
$$

This expression relates the critical value $\lambda_{e}$ to the $\nu_{o}$-measure of the volume of sets of diameter one. Of course, while $\nu_{o}$ must be finite by Assumption 1, it does not have to be a probability measure.

We end this section by giving the proof of Corollary 1.4.

Proof of Corollary 1.4. It follows from Assumption 2 that $\nu_{o}(\mathcal{L}(H))=\nu_{o}(\mathcal{L}(H \cup \partial H))$ and so the statement follows immediately from (4.5).

Remark: In a similar manner, if $\nu_{o}, \nu_{o}^{\prime}$ are both point masses on two different sets having the same volume, then $\lambda_{e}\left(\nu_{o}\right)=\lambda_{e}\left(\nu_{o}^{\prime}\right)$.

\section{$5 \quad$ Examples}

In this section we will briefly review some examples. We will avoid delving into lengthy calculations and discussions and will not give precise references to well known results (e.g. the fractal dimension of the boundary of a von Koch snowflake). Furthermore, although one could in principle just normalize the measure $\mu$ in every example (so that say $\mu\left(\mathcal{A}_{1}\right)=1$ ) this would obviously defeat the purpose. Therefore, the normalizations in the examples are what we consider to be canonical.

It is convenient to have the following proposition which makes it easier to verify condition (1.4). Here, $\overline{\operatorname{dim}}_{B}(\partial H)$ denotes the so called upper box (or Minkowski) dimension of the set $\partial H$. We will not review this concept here but rather refer to Chapter 3 of [7].

Proposition 5.1. Assume that $\nu_{o}$ is concentrated on a finite collection of sets $H_{1}, \ldots, H_{K}$ such that $\max _{k=1, \ldots, K} \overline{\operatorname{dim}}_{B}\left(\partial H_{k}\right)<d$. Then the resulting measure $\mu$ is thin.

Proof. As before, we let $D(x, r)$ denote a ball of radius $r$ centred at $x$. Let $\mathcal{P}_{r}\left(H_{k}\right)$ denote a minimal collection of points such that

$$
\partial H_{k} \subset \bigcup_{x \in \mathcal{P}_{r}\left(H_{k}\right)} D(x, r) .
$$

Since any point $y \in E\left(H_{k}, r\right) \backslash S\left(H_{k}, r\right)$ must be within distance at most $r$ from the boundary, it follows that $y \in D(x, 2 r)$ for some $x \in \mathcal{P}_{r}\left(H_{k}\right)$. Thus,

$$
E\left(H_{k}, r\right) \backslash S\left(H_{k}, r\right) \subset \bigcup_{x \in \mathcal{P}_{r}\left(H_{k}\right)} D(x, 2 r),
$$


and so $\mathcal{L}\left(E\left(H_{k}, r\right) \backslash S\left(H_{k}, r\right)\right) \leq\left|\mathcal{P}_{r}\left(H_{k}\right)\right|(2 r)^{d}$. Let $\alpha$ be such that $\max _{k=1, \ldots, K} \overline{\operatorname{dim}_{B}\left(\partial H_{k}\right)}<$ $\alpha<d$. It follows that for any $r$ small enough we must have that $\left|\mathcal{P}_{r}\left(H_{k}\right)\right| \leq r^{-\alpha}$ for $k=1, \ldots, K$. Therefore,

$$
\mathcal{L}\left(E\left(H_{k}, r\right) \backslash S\left(H_{k}, r\right)\right) \leq r^{-\alpha}(2 r)^{d}=2^{d} r^{d-\alpha},
$$

and so we see that (4.2) is satisfied.

Our first example connects the results of this paper with previous research.

Example 5.2. The fractal ball model.

In this example we shall briefly consider the classical fractal Poisson ball model. Let $\nu(d r)=r^{-d-1} d r$ be a measure on $(0,1 / 2]$ and let $\mu=\mathcal{L} \times \nu$ be the product measure on $\mathbb{R}^{d} \times(0,1 / 2]$. We can view $\mu$ as a measure on $\mathfrak{G}$ by associating a point $(x, r) \in \mathbb{R}^{d} \times(0,1 / 2]$ with the ball (open or closed) $D(x, r)$ of radius $r$. The measure $\mu$ is then taken to be the intensity measure of the fractal ball model, and it is not hard to prove that it is indeed scale invariant and satisfies Assumptions 1, 2 and 3, Furthermore,

$$
\mu\left(\mathcal{A}_{1}\right)=\int_{\mathbb{R}^{d}} \int_{1 / 4}^{1 / 2} I(o \in D(x, r)) \nu(d r) \mathcal{L}(d x)=\int_{1 / 4}^{1 / 2} v_{d} r^{d} r^{-d-1} d r=v_{d} \log 2,
$$

where $v_{d}$ denotes the volume of the unit ball in $\mathbb{R}^{d}$. Thus, by Theorem 1.1 we have that

$$
\lambda_{e}=\frac{d \log 2}{\mu\left(\mathcal{A}_{1}\right)}=\frac{d}{v_{d}} .
$$

Alternatively, we can use the construction of Section 4 with $\nu_{o}$ being unit mass on the unit ball to obtain the same result.

Using Proposition 5.1 we see that $\mu$ is thin, and so by Proposition 1.3, condition (1.4) is satisfied. Thus, it follows from Theorem 1.2 that the Poisson ball model satisfies $\mathcal{C}\left(\lambda_{e}\right)=\emptyset$. This was previously proven in [3] for the case of open balls.

Our next example considers a somewhat more irregular "fundamental" shape than balls. In particular, the shape is non-convex and has a fractal boundary.

Example 5.3. The scale invariant von Koch snowflake model.

An early example of a set with a fractal boundary is the so-called von Koch snowflake (see for instance [7] p. xiv for a construction) which is a subset of $\mathbb{R}^{2}$. We will let $S(x, l, \theta)$ denote such a snowflake with diameter $l$ and orientation $\theta$ compared to some chosen base direction. The boundary of the snowflake is known to have box dimension $\log 4 / \log 3$ while the area of $S(x, l)$ equals $\frac{3 \sqrt{3}}{10} l^{2}$. We can easily turn this into a scale invariant snowflake model by a construction similar to Example 5.2 as we now explain. Let $\Theta$ be uniform measure on $[0,2 \pi)$ and let $\nu(d l)=l^{-3} d l$ on $(0,1]$ (note that $l$ denotes the diameter while $r$ in the previous example was the radius). Then, we let $\mu=\mathcal{L} \times \nu \times \Theta$ be product measure on $\mathbb{R}^{d} \times(0,1] \times[0,2 \pi)$ and to any point $(x, l, \theta) \in \mathbb{R}^{d} \times(0,1] \times[0,2 \pi)$ we associate a snowflake $S(x, l, \theta)$. Then, using $\mu$ as our intensity measure we have constructed a scale invariant Poisson snowflake model. It is easy to check that Assumptions 1, 2 and 3 are all satisfied for this model. 
Furthermore we have that

$$
\begin{aligned}
& \mu\left(\mathcal{A}_{1}\right)=\int_{1 / 2}^{1} \int_{\mathbb{R}^{2}} \int_{0}^{2 \pi} I(o \in S(x, l, \theta)) d \Theta \mathcal{L}(d x) \nu(d l) \\
& =\int_{1 / 2}^{1} \int_{\mathbb{R}^{2}} I(o \in S(x, l, 0)) d \mathcal{L}(x) \nu(d l)=\int_{1 / 2}^{1} \frac{3 \sqrt{3}}{10} l^{2} l^{-3} d l=\frac{3 \sqrt{3}}{10} \log 2
\end{aligned}
$$

and so according to Theorem 1.1 we obtain

$$
\lambda_{e}=\frac{2 \log 2}{\mu\left(\mathcal{A}_{1}\right)}=\frac{20}{3 \sqrt{3}} .
$$

Here, we could also have used $\nu_{o}$ being a rotational invariant measure on snowflakes of diameter 1 centred at $o$.

In addition we can use Propositions 5.1 (the fact that we add rotation to the snowflakes does not change anything) and 1.3 to conclude that $\mu$ satisfies condition (1.4). Then, Theorem 1.2 tells us that for $\lambda=\lambda_{e}, \mathbb{R}^{2}$ is almost surely covered by scale invariant snowfall.

\section{Example 5.4. The Brownian loop soup}

The Brownian loop soup was introduced in [9] and has since received much attention as it is intimately connected to the theory of so-called Schramm-Loewner Evolution (SLE) and Conformal Loop Ensembles $(C L E)$ (see for instance [8]). The Brownian loop measure $\mu^{\text {loop }}$ is a scale invariant measure on Brownian loops in the plane. In this example we will impose a cutoff on the diameter of the loops so that no loop has diameter larger than 1. Furthermore, given a loop $\gamma$ of the loop soup, we define the set $G$ as the complement of the unbounded component created by $\gamma$. The measure $\mu$ is then defined to be the measure on the sets $G$ induced by the outer boundaries of the loops as we just described.

It is known (see for instance [4] Lemma B.8 p. 266) that in this case we have that

$$
\mu\left(\mathcal{A}_{1}\right)=\frac{1}{5} \log 2
$$

from which it follows that

$$
\lambda_{e}=\frac{2 \log 2}{\mu\left(\mathcal{A}_{1}\right)}=10 .
$$

Furthermore, it is proven in [12] Lemma 4 that while the Brownian loop soup in itself is not thin, the measure $\mu$ on the induced sets $G$ is. Therefore, also this example satisfies the conditions of Theorem 1.2 and so $\mathcal{C}\left(\lambda_{e}\right)=\emptyset$.

Acknowledgements: The author would like to thank F. Camia, J. Tykesson and J. Steif for useful comments on an early version of this paper. In addition, the author is grateful towards the anonymous referee for many useful comments and suggestions.

\section{References}

[1] Biermé H. and Estrade A. Covering the whole space with Poisson random balls. ALEA Lat. Am. J. Probab. Math. Stat. 9, (2012), 213-229. 
[2] Broman E. and Camia F. Universal behavior of connectivity properties in fractal percolation models. Electron. J. Probab. 15, (2010), 1394-1414.

[3] Broman E., Jonasson J. and Tykesson J. The existence phase transition for two Poisson random fractal models. Electron. Commun. Probab. 22, (2017), Paper No. 21.

[4] Camia F. Scaling Limits, Brownian Loops and Conformal Fields. Advances in Disordered Systems, Random Processes and Some Application Cambridge University Press (2017), 205-269.

[5] Dekking, F. M. and Meester, R. W. J. On the structure of Mandelbrot's percolation process and other random Cantor sets. J. Statist. Phys. 58, (1990), no. 5-6, 11091126.

[6] Dvoretsky A. On covering a circle with randomly placed arcs Proc. Nat. Acad. Sci. U. S. A. 42, (1956), 199-203.

[7] Falconer K. Fractal Geometry, John Wiley \& Sons, Ltd., (2014).

[8] Lawler G.F. Conformally invariant processes in the plane, Mathematical Surveys and Monographs, 114, American Mathematical Society (2005).

[9] Lawler G.F. and Werner W. The Brownian loop soup. Probab. Theory Relat. Fields, 128, (2004), 565-588.

[10] Lyons R. and Peres Y. Probability on trees and networks Cambridge Series in Statistical and Probabilistic Mathematics, 42 (2016).

[11] Molchanov I. Theory of Random Sets 2nd Edition, Springer-Verlag, (2017).

[12] Nacu S. and Werner W. Random soups, carpets and fractal dimensions. J. Lond. Math. Soc. (2) 83 (2011), no. 3, 789-809.

[13] Shepp L.A. Covering the line with random intervals Z. Wahrscheinlichkeitstheorie und Verw. Gebiete, 23, (1972), 163-170. 